\section{Irradiated shellfish: identification by photostimulated luminescence}

\author{
Maria Concetta Campagna, \\ Maria Teresa Di Schiavi, Grazia Falconi, \\ Francesca Della Verità, Roberta Cavallina \\ Istituto Zooprofilattico Sperimentale \\ delle Regioni Lazio e Toscana, Roma, Italy
}

\section{Abstract}

The irradiation of food is a technology used in the industry to prevent the deterioration of foodstuff in some countries. The European Community legislation states that each Member State must carry out annual checks on the products during commercialisation. The Istituto Zooprofilattico Sperimentale delle regioni Lazio e Toscana (Rome, Italy) has developed and validated the screening method of photostimulated luminescence UNI EN 13751:2009 to identify irradiated shellfish. A total of 30 tests of shellfish samples, consisting of 22 certified as irradiated and 8 not-irradiated samples, were performed. The validation procedure was based on sensitivity and specificity; the compatibility between the screening method and the reference standard EN 13751:2009 was evaluated. Data were processed: $100 \%$ sensitivity and $100 \%$ specificity were obtained. Results obtained in our laboratory were perfectly compatible with the reference standard. For this reason, the method has been validated and proved to be suitable for its intended use.

\section{Introduzione}

L'irraggiamento degli alimenti è una tecnologia utilizzata nell'industria che ha la potenzialità di prevenire il deterioramento delle derrate prolungandone così la shelf-life e migliorandone la salubrità. Gli alimenti vengono sottoposti a dosi ben definite di radiazioni ad alta energia, generalmente raggi gamma, raggi x 0 fasci di elettroni, in grado di alterare l'attività degli enzimi degradativi presenti negli alimenti, di inattivare il materiale genetico delle cellule microbiche, inibendone la suddivisione, $\mathrm{e}$ di devitalizzare eventuali parassiti presenti, ad esempio, in pesci, molluschi e crostacei. Il trattamento con radiazioni ionizzanti è disciplinato dalle direttive quadro 1999/2/CE e 1999/3/CE (Commissione Europea, 1999). La legislazione comunitaria prevede che ciascun alimento e/o ingrediente alimentare trattato con radiazioni ionizzanti, debba riportare in etichetta la dicitura irradiato o trattato con radiazioni ionizzanti. Ciascuno stato membro, inoltre, è tenuto ad effettuare controlli, mediante metodi analitici validati e/o standardizzati a livello europeo, sugli impianti di irraggiamento e sugli alimenti presenti sul mercato al fine di verificare la corretta etichettatura.

L'obiettivo del presente lavoro è stato quello di mettere a punto e validare il metodo fisico di screening in luminescenza otticamente stimolata (photostimulated luminescence, PSL) per la rivelazione di molluschi e crostacei trattati con radiazioni ionizzanti presso l'Istituto Zooprofilattico Sperimentale delle regioni Lazio e Toscana, Roma.

\section{Materiali e Metodi}

Il metodo PSL (EN 13751:2009) (UNI, 2009) si basa sulla misura della luce emessa dai particolati minerali presenti naturalmente nel campione che, se precedentemente irradiati, emettono luce in seguito a stimolazione ottica. Si misura l'intensità dell'emissione luminosa in termini di conteggi per unità di tempo (conteggi/60 secondi). Il risultato viene confrontato con due soglie di riferimento, T1 (soglia minore) e T2 (soglia maggiore). Per segnali superiori alla soglia T2 il campione viene definito irradiato, ma deve essere comunque classificato correttamente attraverso uno dei metodi di conferma Comitato Europeo di Normazione. Segnali inferiori alla soglia T1 suggeriscono che il campione non ha subito un trattamento con radiazioni ionizzanti ed è
Correspondence: Maria Concetta Campagna, Istituto Zooprofilattico Sperimentale delle Regioni Lazio e Toscana, via Appia Nuova 1411, 00178 Roma, Italy.

Tel. +39.067.9099323 - Fax: +39.067 .9340724 .

E-mail: mariaconcetta.campagna@izslt.it

Key words: Photostimulated luminescence, Shellfish, Irradiated food, UNI EN 13751:2009.

Received for publication: 9 May 2013.

Revision received: 5 November 2013.

Accepted for publication: 7 November 2013.

This work is licensed under a Creative Commons Attribution 3.0 License (by-nc 3.0).

(C) Copyright M.C. Campagna et al., 2014

Licensee PAGEPress, Italy

Italian Journal of Food Safety 2014; 3:1653

doi:10.4081/ijfs.2014.1653

perciò classificato come non irradiato. Valori intermedi alle due soglie esprimono un risultato incerto che deve essere correttamente classificato mediante un ulteriore metodo standardizzato.

Sono stati acquistati, presso punti vendita al dettaglio, n.8 campioni: mitili (Mytilus chilensis), vongole (Paphia undulata), capesante (Argopecten purpuratus), gamberi rosa (Parapenaeus longirostris), gamberi argentini (Hymenopenaeus muelleri), scampi (Nephrops norvegicus), mazzancolle (Penaeus vannamei) e granchio (Cancer magister). Essi sono stati dapprima analizzati in PSL per verificarne la negatività $\mathrm{e}$, in seguito, inviati all'IZS della Puglia e della Basilicata per essere irraggiati ciascuno a tre dosi distinte: 0,5, 3 e 5 KGy. Solo nel caso del granchio l'irraggiamento è avvenuto ad una dose singola di 1 KGy. I n.22 campioni irraggiati sono stati, quindi, sottoposti all'analisi in PSL per rilevare l'avvenuto trattamento.

L'analisi è stata condotta mediante analizzatore con sistema di luminescenza pulsata stimolata (Scottish Universities Environmental Research Centre pulsed photostimulated luminescence, SUERC PPSL) sui campioni sgusciati. Le soglie di riferimento sono state impostate a $1000 \mathrm{c} / 60 \mathrm{~s}$ (T1) e 4000c/60s (T2), in accordo con

Tabella 1. Risultati dell'analisi in luminescenza otticamente stimolata di crostacei e molluschi.

\begin{tabular}{lcccccccc} 
Dose (KGy) & Vongole & Mitili & Gamberi rosa & Capesante & Scampi & Gamberi & Marmancolle & Granchio \\
0 & NI & NI & NI & NI & NI & NI & NI \\
0,5 & IRR & IRR & IRR & IRR & IRR & IRR & IRR \\
\hline 1 & - & - & - & - & - & - & - & IRR \\
3 & IRR & IRR & IRR & IRR & IRR & IRR & IRR \\
\hline 5 & IRR & IRR & IRR & IRR & IRR & IRR & IRR & - \\
\hline
\end{tabular}

NI, non irraggiato; IRR, irraggiato. 
la Norma di riferimento. La conferma analitica dei campioni risultati irradiati alla misura in PSL è stata eseguita mediante metodo fisico di termoluminescenza (EN 1788:2001; Commissione Europea, 2001). La validazione del metodo è stata eseguita determinandone la sensibilità e la specificità; è stata valutata la compatibilità del metodo con la norma di riferimento UNI EN 13751:2009 (UNI, 2009).

\section{Risultati}

In Tabella 1 sono riportati gli esiti dell'analisi in PSL dei campioni. I risultati delle prove si sono dimostrati in accordo con l'effettivo stato di irraggiamento dei campioni; sono stati ottenuti valori di sensibilità e specificità del 100\%.

\section{Discussione e Conclusioni}

Tutti i campioni esaminati sono stati correttamente identificati. I risultati ottenuti presso il nostro laboratorio si sono dimostrati perfettamente compatibili con quelli contenuti nella citata Norma di riferimento. Per tale motivo il metodo è stato validato e ritenuto idoneo per l'utilizzo previsto.

\section{Bibliografia}

Commissione Europea, 1999. Direttiva 1999/2/CE del Parlamento europeo e del Consiglio del 22 febbraio 1999 relativa al ravvicinamento delle legislazioni degli Stati membri concernenti gli alimenti e i loro ingredienti trattati con radiazioni ionizzanti. In: Rivista Ufficiale, L 66, 13/03/1999.

Commissione Europea, 2001. Foodstuffs. Thermoluminescence detection of irradiated food from which silicate minerals can be isolated. Disponibile al sito: http://ec.europa.eu/food/food/biosafety/irra diation/1788-2001_en.pdf

UNI, 2009. UNI EN 13751:2009 Norm. Prodotti alimentari - Ricerca di alimenti irraggiati con I utilizzo di luminescenza fotostimolata. Istituto Italiano per l'Unificazione ed., Milano, Italy. 